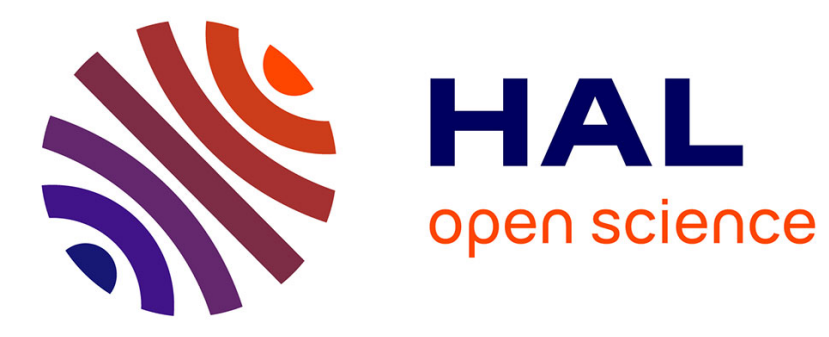

\title{
Quasi-modal analysis of segmented waveguides
}

André Nicolet, G Demésy, F Zolla, Benjamin Vial

\section{To cite this version:}

André Nicolet, G Demésy, F Zolla, Benjamin Vial. Quasi-modal analysis of segmented waveguides. 2014 IEEE Conference on Antenna Measurements \& Applications (CAMA), Nov 2014, Antibes Juanles-Pins, France. 10.1109/CAMA.2014.7003327 . hal-01340772

\section{HAL Id: hal-01340772 \\ https://inria.hal.science/hal-01340772}

Submitted on 1 Jul 2016

HAL is a multi-disciplinary open access archive for the deposit and dissemination of scientific research documents, whether they are published or not. The documents may come from teaching and research institutions in France or abroad, or from public or private research centers.
L'archive ouverte pluridisciplinaire HAL, est destinée au dépôt et à la diffusion de documents scientifiques de niveau recherche, publiés ou non, émanant des établissements d'enseignement et de recherche français ou étrangers, des laboratoires publics ou privés. 


\title{
Quasi-modal analysis of segmented waveguides
}

\author{
A. Nicolet, G. Demésy, F. Zolla, B. Vial \\ Institut Fresnel \\ Aix-Marseille Université, CNRS, Ecole Centrale de Marseille \\ Marseille, France \\ andre.nicolet@fresnel.fr
}

\begin{abstract}
In the present paper, we show that it is possible to use a periodic structure of disconnected elements (e.g. a line of rods) to guide electromagnetic waves, in the direction of the periodicity. To study such segmented waveguides, we use the concept of quasimodes associated to complex frequencies. The numerical determination of quasimodes is based on a finite element formulation completed with Perfectly Matched Layers (PMLs). These PMLs lead to non Hermitian matrices whose complex eigenvalues correspond to quasimode frequencies. Using Floquet-Bloch theory, a numerical model is set up that allows the spectral study of structures that are both open and periodic. With this model, we show that it is possible to guide electromagnetic waves on significant distances with very limited losses.
\end{abstract}

\section{INTRODUCTION}

In this paper, we show that it is possible to efficiently guide electromagnetic waves with discontinous structures, i.e. with a periodic chain of disconnected elements usually named segmented waveguides [1]. Traditional waveguides such as optical fibres or metallic waveguides are usually presented as structures with a cross section invariant along one direction, e.g. $x$-axis. The theoretical and numerical studies consist in determining propagation modes that are electromagnetic fields with components in the form $U(y, z) e^{-i(\omega t-\alpha x)}$ where $U(y, z)$ is a function on the cross section, $\omega \in \mathbb{R}$ is the pulsation and $\alpha \in \mathbb{R}$ is the propagation constant. Nevertheless, it may be useful to generalize this concept of propagation mode to complex $\omega=\omega^{\prime}+i \omega^{\prime \prime}(\omega \in \mathbb{C}, \alpha \in \mathbb{R})$ or complex $\alpha=\alpha^{\prime}+i \alpha^{\prime \prime}(\omega \in \mathbb{R}, \alpha \in \mathbb{C})$. In this case, the propagation of the mode is accompanied by an exponential decrease in time or along the propagation direction due to the fact that the guided wave is not well confined in the guide that is therefore a lossy guide. We consider here lossless materials and the losses do occur because of the configuration of the guide (open waveguides) and not because the materials are dissipative. The corresponding "modes" are named quasimodes or leaky modes. They may seem uninteresting from a practical point of view but in fact the imaginary part of $\omega$ may be small enough to transport waves on significant distances.

We will therefore consider segmented waveguides and look for modes and, most probably, quasimodes with losses weak enough.

\section{QUASIMODES}

In order to study the segmented waveguides, we are now led to determine the quasimodes but they are not just a generalization of modes to complex frequencies/propagation constants and special techniques have to be used in order to compute them properly.

Modes are solutions to the wave equation without sources and these solutions have a finite power. From a mathematical point of view, it means that they are elements of a suitable Hilbert space whose norm is related to the power of the physical field. It can be shown that the quasimodes can not be elements of this Hilbert space because their norm/power is necessarily infinite. To show this heuristically, consider classical waveguides invariant along $x$ with modes that are fields with components in the form $U(y, z) e^{-i(\omega t-\alpha x)}$. For the quasimodes, we consider, in this section, the $(\omega \in \mathbb{R}, \alpha \in \mathbb{C})$ case. In the $y z$-plane, outside of the guide (i.e. the region where the media are different from the surrounding medium), we consider that the medium is isotropic homogenous and characterized by permittivity $\varepsilon_{\infty} \in \mathbb{R}$. The components of a quasimode satisfy a Helmholtz equation $\Delta U+k_{\infty}^{2} U=0$ with $k_{\infty}^{2}=k_{o}^{2} \varepsilon_{\infty}-\alpha^{2}$ and $k_{o}=\omega / c$. The solutions in cylindrical coordinates can be written as a Fourier-Bessel expansion $U(\rho, \phi)=\sum_{n \in \mathbb{Z}} c_{n} H_{n}^{(1)}\left(k_{\infty} \rho\right) e^{i n \varphi}$. The asymptotic behavior for large $\rho$ is $U(\rho, \varphi)=\sigma(\varphi) e^{i k_{\infty} \rho} / \sqrt{\rho}+O\left(\rho^{-3 / 2}\right)=$ $\sigma(\varphi) e^{i k_{\infty}^{\prime} \rho} e^{-k_{\infty}^{\prime \prime} \rho} / \sqrt{\rho}+O\left(\rho^{-3 / 2}\right)$. Since $k_{o}, \varepsilon_{\infty}$ are real, $k_{\infty}=k_{\infty}^{\prime}+i k_{\infty}^{\prime \prime}$ is complex with $\alpha^{\prime} \alpha^{\prime \prime}=-k_{\infty}^{\prime} k_{\infty}^{\prime \prime}$ (imaginary part of $k_{\infty}^{2} / 2$ ). We have $k_{\infty}^{\prime}>0$ (outgoing wave condition), $\alpha^{\prime}>0$ (propagation towards positive $x$ ), and $\alpha^{\prime \prime}>0$ (leaky mode decreasing along the propagation direction) that give $k_{\infty}^{\prime \prime}<0$, i.e an exponential divergence in the cross section with $\rho \rightarrow \infty$.

\section{FE AND PMLS}

Nowadays, the Finite Element (FE) method is an effective method to compute the behaviour of electromagnetic waves in photonic devices. It provides a tool that is extremely versatile and accurate at a reasonable computational cost. From now, we consider the case of complex frequencies $(\alpha \in \mathbb{R}, \omega \in$ $\mathbb{C})$. An important progress has been made by the introduction of Perfectly Matched Layers (PMLs) that are used for the reflectionless truncation of infinite domains [2]. Moreover, the PMLs are the theoretical and numerical tool that we need to compute the quasimodes, specially for the correct computation of the imaginary parts of the frequencies associated to leakages [3]. The role of the PMLs is to rotate the continuous spectrum in the complex plane [4] in order to unveil the quasimodes (by providing a non-Hermitian extension of the operator associated to the initial problem). A practical way to design such PMLs 
is to consider Transformation Optics: PMLs may be obtained by applying a complex-valued stretch to the coordinates and then computing the resulting equivalent materials [5].

\section{Periodic WaVEguides}

The last feature required to study the segmented waveguides is to take into account their periodic structures. In the case of translational invariance, only the cross section has to be taken into account and this reduces the geometric dimension of the problem, e.g. 3D to $2 \mathrm{D}$. Here we are interested in more general case where the waveguide is periodic along the propagation direction. There is no more dimension reduction but the numerical problem can be set up on a single cell of the periodic problem via Floquet-Bloch quasiperiodic boundary conditions (FBC) [6]. The quasiperiodicity condition is determined by the size of the cell together with the value of the propagation constant $\alpha$ (real and a priori given, the problem is to find the various associated $\omega$ ). A numerical model is set up, using FE, PMLs, and FBC, that allows the spectral study of structures that are both open and periodic. This model is the tool we are using to investigate the quasimodes of the segmented waveguides.

\section{RESUlts: Finite ELEMENT MODELING OF PERIODICALLY SEGMENTED WAVEGUIDES.}

In this section, we consider particular waveguides made of parallel dielectric rods in the visible range.

\section{A. Chain of infinitely long cylinders.}

In this first part, we consider the 2D case (see Fig. 1). Our study begins with the determination of the quasimodes by considering a spectral problem in a single cell of the open and periodic waveguide shown in Fig. 1a. We use the FE method implemented in GetDP freeware [7] together with PMLs and FBC. Figure 2 represents the dispersion diagram obtained

(a) infinite chain - spectral problem

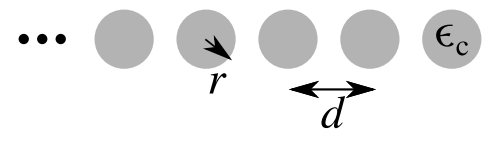

(b) finite chain - with source
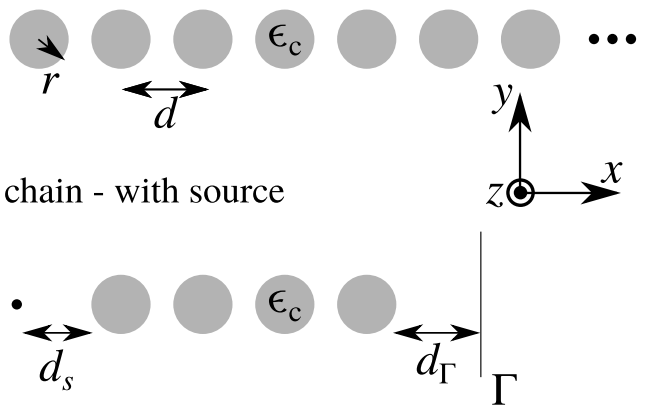

Fig. 1. Schematic of the two problems studied in this paper. (a) Spectral problem: Quasi-modes of an infinite chain of periodically arranged dielectric cylinders of radius $r$, relative permittivity $\epsilon_{c}$, and periodicity $d$. (b) Direct problem: Transmission of a finite chain (4 "periods" are represented here) enlighten by a monopole antenna.

with $\alpha$ spanning the first Brillouin zone in both polarization cases. Figure $2 \mathrm{c}$ shows the corresponding transmission of the finite guide (see Fig. 1b using 80 rods). Figure $3 \mathrm{a}$ shows the complex eigenfrequencies for a given value of the propagation constant. Figure $3 \mathrm{~b}$ to $3 \mathrm{f}$ shows $\operatorname{Re}\left\{E_{z}\right\}$ for some of the corresponding eigenmodes. Points close to the real axis (e.g. (b) and (d)) are leaky modes (intrinsic to the waveguide, i.e. independent of the PML parameters [3]). The other points (c), (e) and (f) correspond to the discretization of the continuous spectrum. These points depend strongly on the parameters of the PMLs and have been discarded in Fig. 2. Points (c) and (e) represent modes with moderate losses. They can be interpreted as quasi-plane waves having significant values in the non PML region. Finally, point (f) exhibits very important losses and corresponds to a field lying mainly within the PMLs. Figure 4 shows the behavior of the field for various wavelengths in the case shown in Fig. 1b (finite chain with EM source and 80 rods, E//-polarization case). The case $\lambda=620 \mathrm{~nm}$ corresponds to a leaky mode with significant losses. The case $\lambda=700 \mathrm{~nm}$ corresponds to a mode in the first gap shown in Figs $2 \mathrm{~b}$ and 2c. The case $\lambda=800 \mathrm{~nm}$ corresponds to a leaky mode with very small losses. The structure can be considered as a good waveguide in this case.

\section{B. Chain of cylinders of finite length.}

We perform the same type of calculations in the case of cylinders of finite length $l=6 r$ (along $z$ ). In this more realistic case, we are dealing with a 3D problem leading to large scale computation involving vector fields (relying on the use of edge elements). Figure 5 shows the dispersion diagram (directly for all possible polarizations). It can be observed that the 2D dispersion diagrams shown in Figs $2 \mathrm{a}$ and $2 \mathrm{~b}$ are very similar to the $3 \mathrm{D}$ one, suggesting that the finite length has a limited impact on the real part of the eigenfrequencies. Figure 6 shows

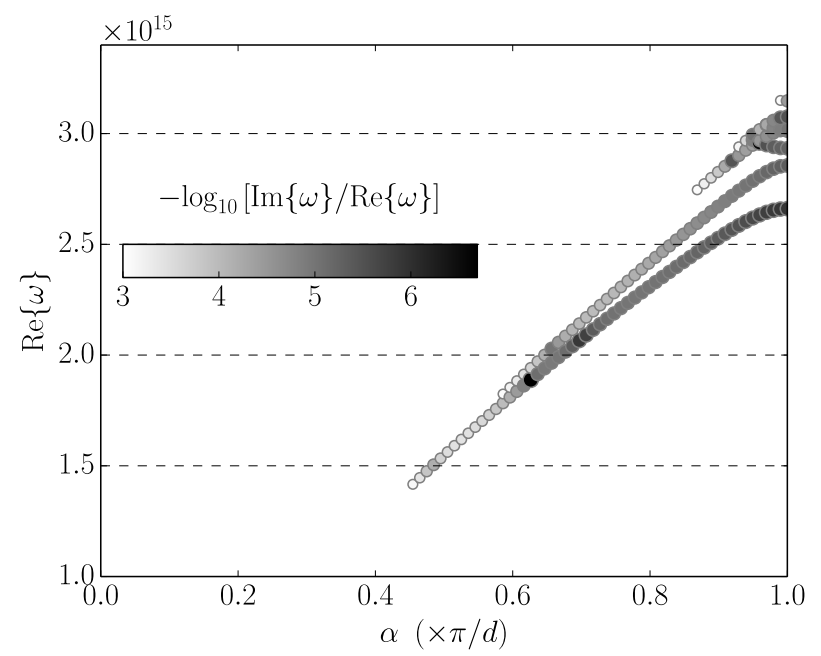

Fig. 5. Dispersion diagram for the quasimodes ( $\omega$ in $\mathrm{rad} / \mathrm{s}$ ) of an infinite chain of dielectric cylinders of finite size $l=6 r$, with $d=300 \mathrm{~nm}, r=125 \mathrm{~nm}$ and $\epsilon_{c}=2.25$ (3D vector model with edge elements).

the real part of the electric field in the $x y$ plane for a finite chain of finite length cylinders with a dipole source at $750 \mathrm{~nm}$. 

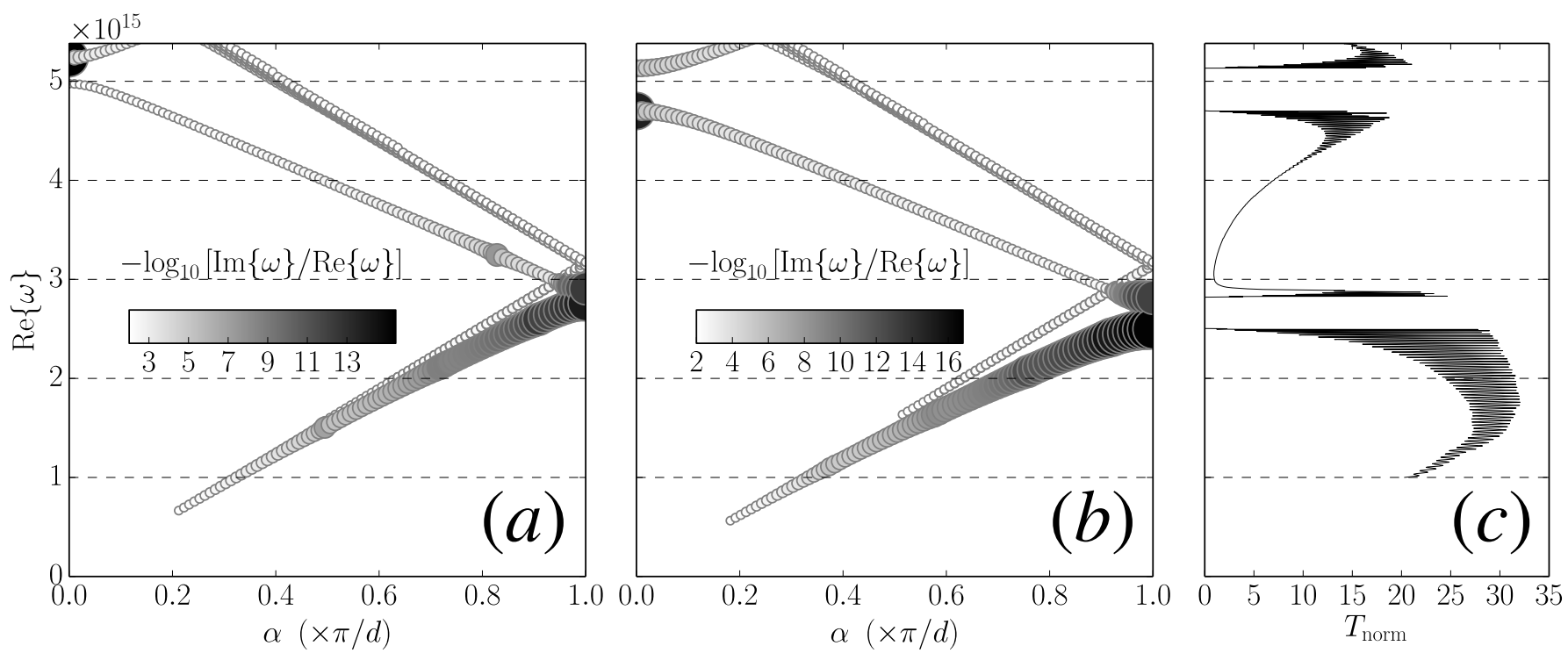

Fig. 2. Dispersion diagram for the quasimodes ( $\omega$ in rad/s) of an infinite chain of dielectric infinitely long cylinders with $d=300 \mathrm{~nm}, r=125 \mathrm{~nm}$ and $\epsilon_{c}=2.25$ for $\mathrm{H} / /$-polarization $\left(H_{z} \neq 0\right)$ case (a) and $\mathrm{E} / /$-polarization $\left(E_{z} \neq 0\right)$ case (b). The greyscale and the size of the dots (the larger, the less losses) reflects the real part/imaginary part ratio of the quasimodes. Normalized transmission (see Fig. 1: Ratio of Poynting fluxes across $\Gamma$ with/without waveguide) spectrum of a 80-cylinders long chain corresponding to polarization case (b).
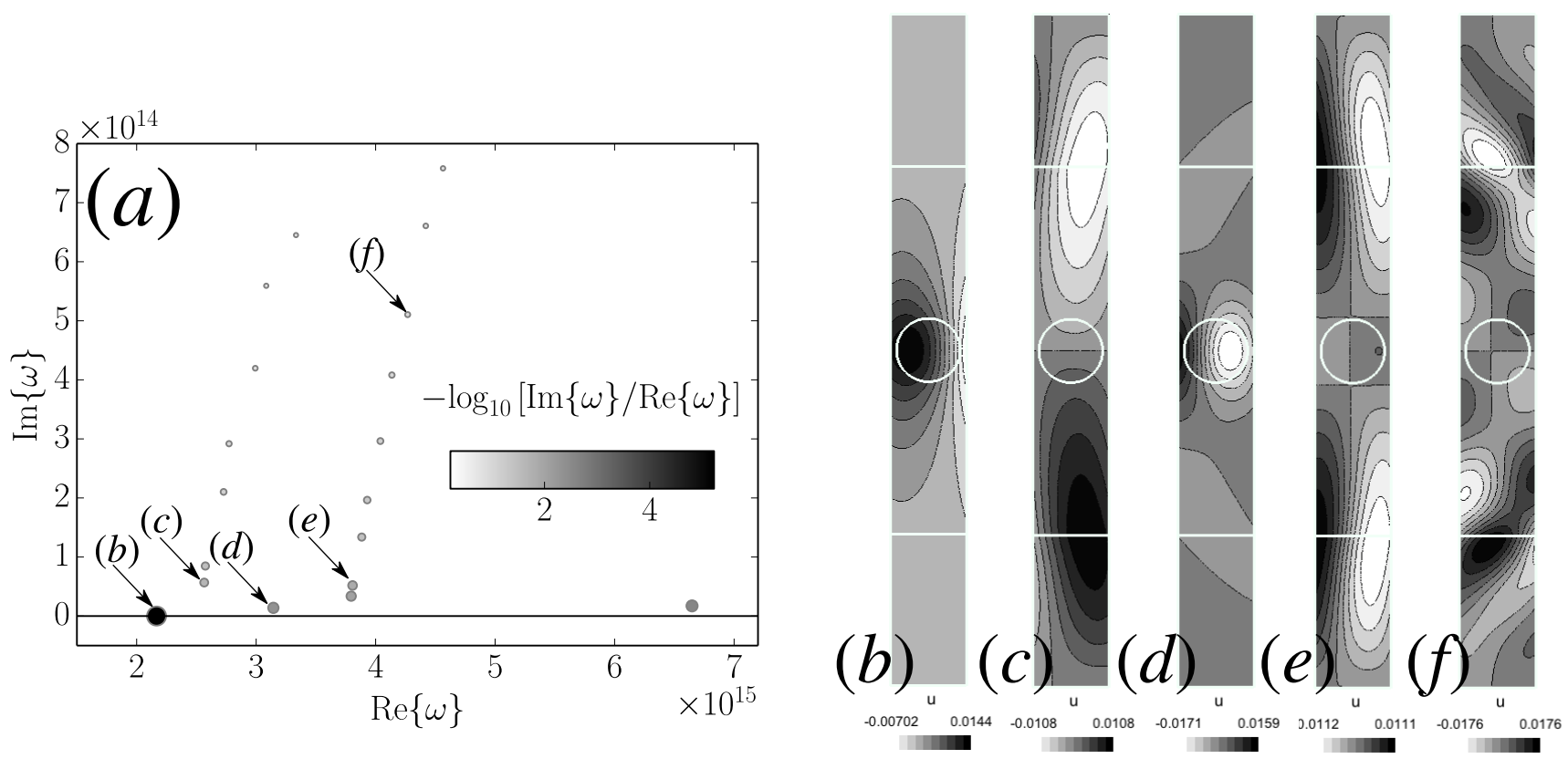

Fig. 3. (a) Distribution of the complex frequencies for the propagation constant $\alpha=0.8 \pi / d$ for the $2 \mathrm{D}$ case shown in Fig. $2 \mathrm{~b}$. Isovalue maps (b) to (f) represent $\operatorname{Re}\left\{E_{z}\right\}$ for some of the corresponding eigenmodes shown in (a).

\section{CONCLUSION}

We have shown, using the concept of propagating quasimode (leaky modes) that very simple periodic structures made of identical elements can be efficient waveguides. Such structures would be more convenient to build than traditional photonic crystal waveguides. Work in progress include the experimental verification and the study of other settings.

\section{REFERENCES}

[1] H. Němec, P. Kužel, J.-L. Coutaz, J. Čtyrokỳ, “Transmission properties and band structure of a segmented dielectric waveguide for the terahertz range", Optics communications, vol. 273, pp. 99-104, 2007.

[2] J. Berenger "A perfectly matched layer for the absorption of electromagnetic waves", Journal of Computational Physics, vol. 114, 2, pp. 185-200, 1994.

[3] B. Vial, F. Zolla, A. Nicolet, M. Commandré, "Quasimodal expansion of electromagnetic fields in open two-dimensional structures", Phys. Rev. A, vol. 89, 023829, 2014 


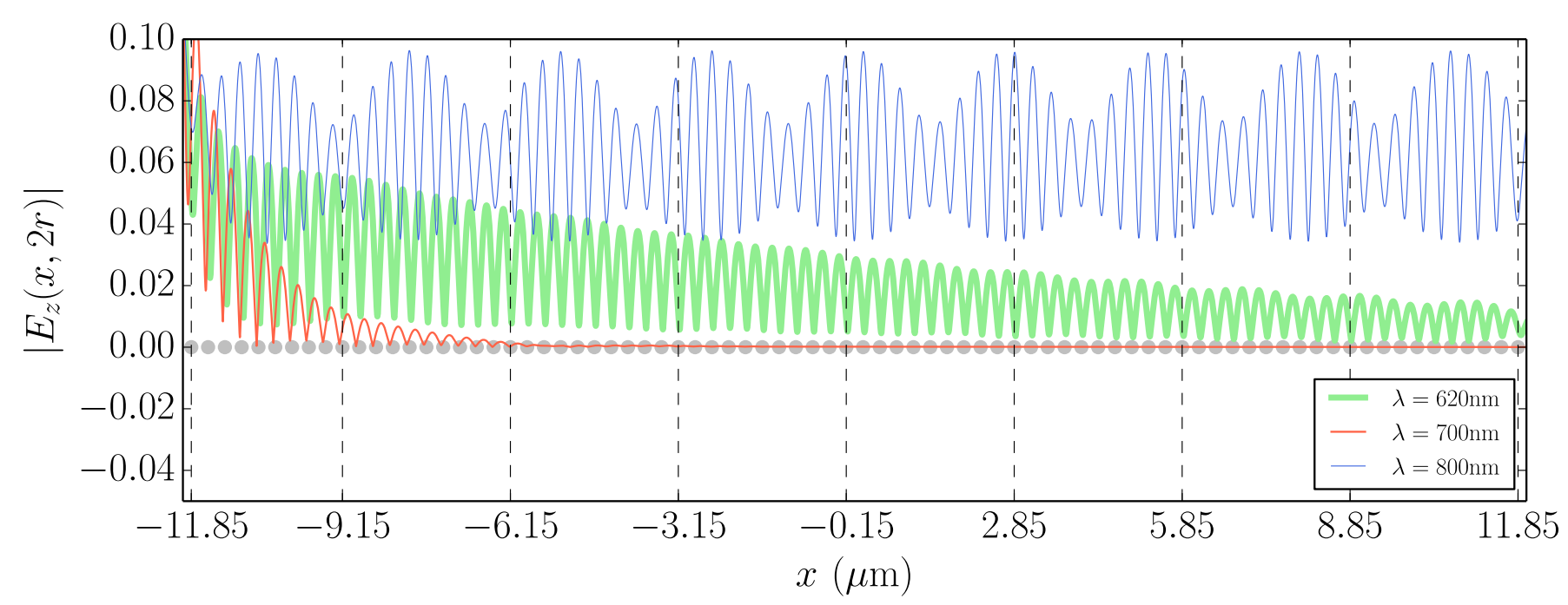

Fig. 4. Norm of the electric field $\left|E_{z}(x, 2 r)\right|$ calculated on a line parallel to the guide (at a distance $r$ above the waveguide), for different monopole wavelengths : $620 \mathrm{~nm}$ in thick (green, color online) solid line, $700 \mathrm{~nm}$ in normal (red) solid line and $800 \mathrm{~nm}$ in thin (blue) solid line.

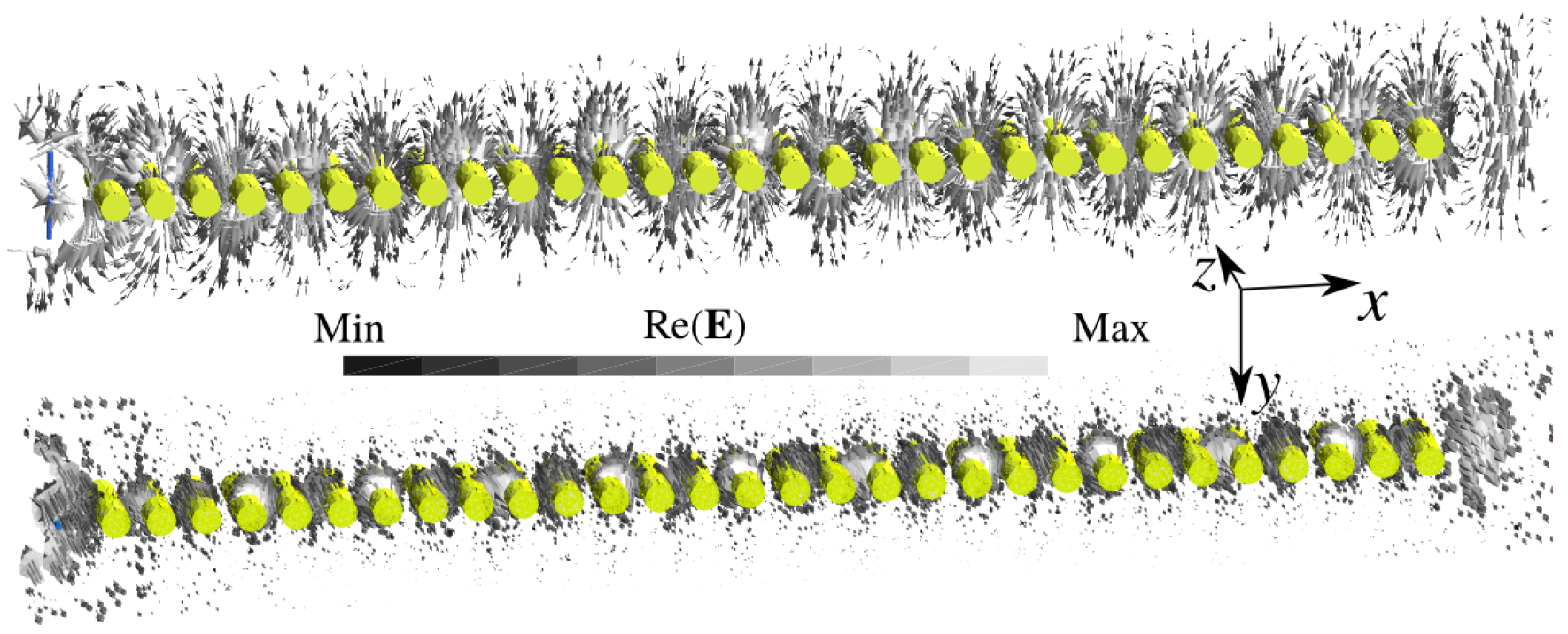

Fig. 6. Finite chain of 30 cylinders of finite length $l=6 r$, with $d=300 \mathrm{~nm}, r=125 \mathrm{~nm}$ and $\epsilon_{c}=2.25$ enlighten by a dipole antenna at $750 \mathrm{~nm}$. Real part of the electric field in the $x z$ plane. Two possible orientations - along $y$ (a) or $z$ (b) - of the dipole antenna leads to the excitation of one of the two modes with $\operatorname{Re}\{\omega\}=2.5 \times 10^{15} \mathrm{rad} / \mathrm{s}$ in Fig. 5 .

[4] F. Olyslager "Discretization of Continuous Spectra Based on Perfectly Matched Layers", SIAM Journal on Applied Mathematics, vol. 64, 4, pp. 1408-1433, 2004.

[5] Y. Ould Agha, F. Zolla, A. Nicolet, S. Guenneau, "On the use of PML for the computation of leaky modes - An application to microstructured optical fibres", COMPEL, vol. 27, 1, pp. 95-109, 2008.

[6] A. Nicolet, S. Guenneau, C. Geuzaine, F. Zolla "Modelling of electromagnetic waves in periodic media with finite elements", Journal of Computational and Applied Mathematics, vol. 168, 1, pp. 321-329, 2004.

[7] P. Dular, C. Geuzaine, F. Henrotte, W. Legros "A general environment for the treatment of discrete problems and its application to the finite element method", IEEE Transactions on Magnetics, vol. 34, 5, pp. 3395-3398, 1998. 